\title{
A New Design Solution for Aircraft Wheels that Reduces Overpressure in the Tire while Retaining its Absorption Power and its Dimensions
}

\author{
Dalibor Petrović1 - Marjan Dodić1 - Nenad Kapor2,* \\ 1 University of Defence, Military Academy, Serbia \\ ${ }^{2}$ Megatrend University, Faculty of Civil Aviation, Serbia
}

It is well-known that high working overpressure in a tire adversely affects its service life, while a lower overpressure requires larger tire dimensions and weight. The goal of this research is to find the way to decrease the overpressure in a standard tire in order to increase its durability, while preserving or increasing its load-capacity and retaining the same geometric characteristics. This paper presents a new construction solution that consists of a standard (outer) tire and a smaller (inner) tire inserted in the standard one. This set of tires creates a unique kinetic energy absorption system that occurs when the aircraft lands in direct contact with the runway. During the aircraft hard landing, a large deformation of the tire occurs, i.e., the tire surface is drawn inward both in the cross-section and in the longitudinal section. In this case, the whole weight of the airplane is directed on the tire-shoulder, meaning that the actual contact surface is small. In the new solution, the inner tire prevents the standard tire from drawing inward and keeps it in contact with the runway during tire deflection. A comparative analysis of the change in deflection, track width, and stress distribution of a standard tire and a set of tires in the new solution is performed. It was shown that the new construction solution increases the tire track width by about $8 \%$ and, therefore, the greater contact area between the tires and the runway. In addition, tire stress is decreased by about $40 \%$; therefore, it has higher tire durability, as well as the ability to absorb more kinetic energy by $56 \%$ compared to a standard tire.

Keywords: aircraft tire, finite element method, tire design, kinetic energy, absorption system

\section{Highlights}

- A new construction solution that consists of a standard (outer) tire and a smaller (inner) tire inserted in the standard one was proposed.

- The inner tire prevents the standard tire from drawing inward and it keeps it in contact with runway during tire deflection.

- The idea was to ensure that the tread of the tire is in full contact with the runway during the hard aircraft landing.

- The results of performed simulation show that the new construction solution increases the tire track width by about $8 \%$; therefore, a greater contact area between the tires and the runway is observed.

- The presented solution ensures lower tire stress by about $40 \%$; therefore, higher tire durability, as well as the ability to absorb more kinetic energy by $56 \%$ compared to the standard tire, is possible.

\section{O INTRODUCTION}

The development of aviation has led to high-speed aircraft that require thinner wings. The thinner wings required the reduction of tire dimensions; the tire width has to be reduced to fit into the wing structure. In order to ensure sufficient kinetic energy absorption, such thin tires are exposed to high overpressures.

In this scope, it necessary to analyse the primary role of an aircraft tire, that is, the kinetic energy absorption of the impact during landing. The amount of kinetic energy to be absorbed at the moment the tire contacts the runway depends on the landing. Landing is the most sensitive phase of flight, i.e., tire behaviour at the moment when the aircraft touches the runway depends on the piloting technique, aircraft flight parameters, runway condition, atmospheric conditions, and aircraft weight [1]. This paper analyses the tire behaviour under assumption that the landing is performed by standard piloting technique in a quiet atmosphere. In addition, it is assumed that the tire and runway contact is instantaneous and at right angle. In this case, the tire has the highest deflection, inflation, and stresses in its structure.

The goal of this study is to find a solution to decrease the overpressure in a standard tire in order to increase its durability, while preserving or increasing its load-capacity and keeping the same geometric characteristics. In this scope, recent construction solutions of a vehicle's tire from the car industry were analysed, as this is the most advanced area in sense of new ideas for improving the characteristics of a pneumatic. One recent constructive solution is a new non-pneumatic mechanical elastic tire [2], in which elastic rings and hinges are used instead of air overpressure, in order to improve the tire performance. However, this non-pneumatic solution performed well only for smaller velocities, while for 
larger velocities, the pneumatic solution showed better performance. For this reason, this study is directed to the construction of a pneumatic tire.

During the aircraft hard landing, a large deformation of the tire occurs, as was shown in the study [3]. More precisely, the tire surface is drawn inward in the cross-section and in the longitudinal section. In this case, the whole weight of the airplane is directed on the tire-shoulder.

Recent efforts have been focussed on the analysis of the standard tire shape and its improvement [4]). In contrast, no consideration has been given to alternative solutions for the wheel structure in the main landing gear in order to improve the tire characteristics.

Considering that mass is the most important limiting factor in aviation, the idea of modifying the aircraft wheel assembly to achieve pressure reduction and to increase tire kinetic energy absorption, while retaining the dimensions of the aircraft tire, might seem to be contradictory and impossible at a first glance. It may be the reason that there were no research in this direction present in the literature thus far.

The complex conditions of contact [5], large deformations, as well as geometric and material nonlinearities, lead to the conclusion that the aircraft tire can be analysed only by using the finite element method. In the analysis of aircraft tires, the use of the finite element method has led to the development of different modelling approaches for aircraft tires, in order to predict their behaviour under large changes in overpressures and stresses that occur at the moment of contact between the tire and the runway [6].

Korunović et al. consider the symmetrical case of airplane landing [7] (i.e., it is assumed that both tires simultaneously contact the runway) in order to analyse the deformations that occur in a tire during this case of landing. Differently from [8], this study analyses only the moment of landing, i.e., the moment of contact of the tire with the runway. As the contact time is very short, it is assumed that at this moment there is no increase in temperature in the tire, which is contrary to studies [9] and [10], in which the thermomechanical behaviour of an aircraft tire in critical situations is studied.

Therefore, the idea of this study is to ensure tire deflection during the aircraft landing is such that the tire tread is in full contact with runway. This paper proposes a new construction solution that consists of a standard (outer) tire and a smaller (inner) tire inserted in the standard one. The inner tire prevents the standard one from drawing inward it keeps it in contact with runway during tire deflection.
For the successful modelling of this construction solution, it is necessary to define the geometry of the tires, but also to model the material as realistically as possible. For this scope, the experimental results from the testing of materials were used to obtain input data that are necessary to define their characteristics as accurately as possible, which is of great importance when applying the finite element method. The Mooney-Rivlin model was used for tread modelling, as suggested in [11].

As in [12], this study uses experimentally obtained results for rubber definition. The only difference with [12] is that the compression experiment was not performed. Instead, the biaxial tensile test, pure shear test, and volume compression test were used (Section $4)$.

\section{PROBLEM DEFINITION AND ITS SOLUTION}

Aircraft tires bear the weight of the aircraft only through overpressure, and their construction has not changed much. The only change is that aircraft tubeless tires are used, instead of tires with inner tires. Although it is well known that working pressure affects tire life, the following problem has not been considered: how to reduce tire overpressure for the same load-capacity and the same geometric characteristics.

This problem is difficult to solve with a standard wheel structure, since a lower overpressure requires a larger tire. However, a larger tire has many imperfections, and the most important one (besides the weight gain) is the space that a larger tire occupies. Therefore, given the fact that reducing the pressure in a tire would extend the life of the tire, the question is how to do this without disrupting the existing geometry?

Tire contact with the runway causes tire deflection (Fig. 1). In the case of larger deflections, there is deformation on the tire, both in the cross-section and in the longitudinal section of the tire (Fig. 2), i.e., the tire surface is drawn inward.

To prevent the tire from drawing inward, a smaller (inner) tire is placed inside the standard (outer) tire and is mounted in the inner torus of the outer tire. The smaller (inner) tire is positioned so that its outer torus is at a certain distance $\Delta$ from the tip of the inner torus of the outer tire (Fig. 3).

This distance $\Delta$ is defined by the deformation of the outer tire, that is, the outer torus of the smaller (inner) tire is located at the distance at which the standard (outer) tire starts to draw inward. The position of the inner tire is such that it prevents the 
outer tire from twisting inward and forces the outer tire to expand to the side [6]. In this way, the track width of the outer tire is increasing, i.e., its contact surface is larger.

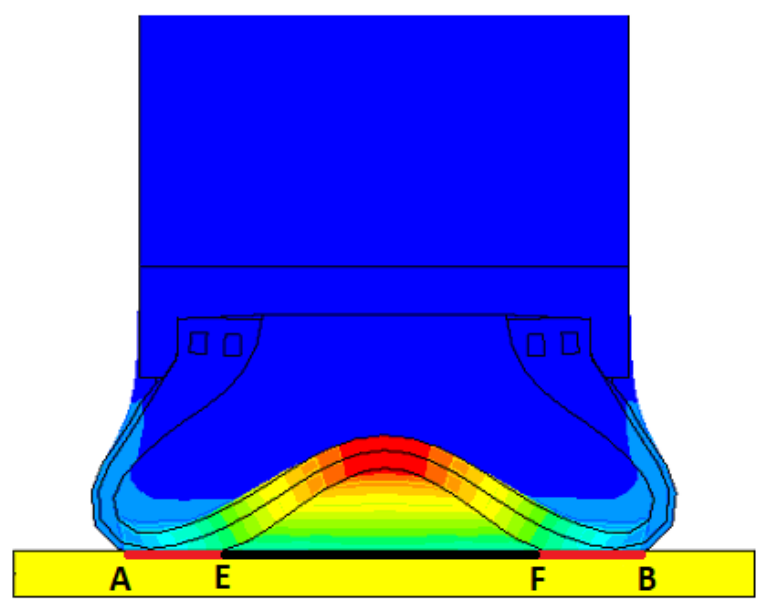

Fig. 1. Tires cross-section at deflection $h=70 \mathrm{~mm}$; $A, B$ : the outer boundaries of the contact surface; $E, F$ : the inner boundaries of the contact surface

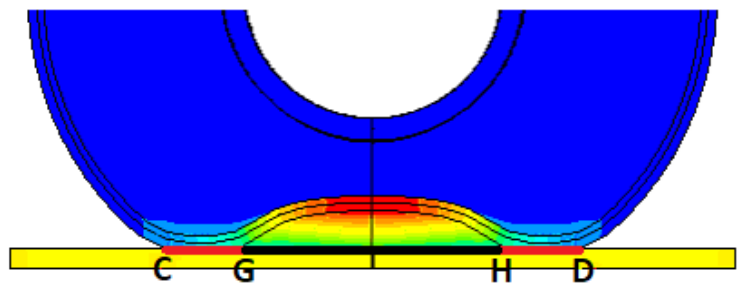

Fig. 2. Tire's longitudinal -section at deflection $h=70 \mathrm{~mm}$; C, D: the outer boundaries of the contact surface; $\mathrm{G}, \mathrm{H}$ : the inner boundaries of the contact surface

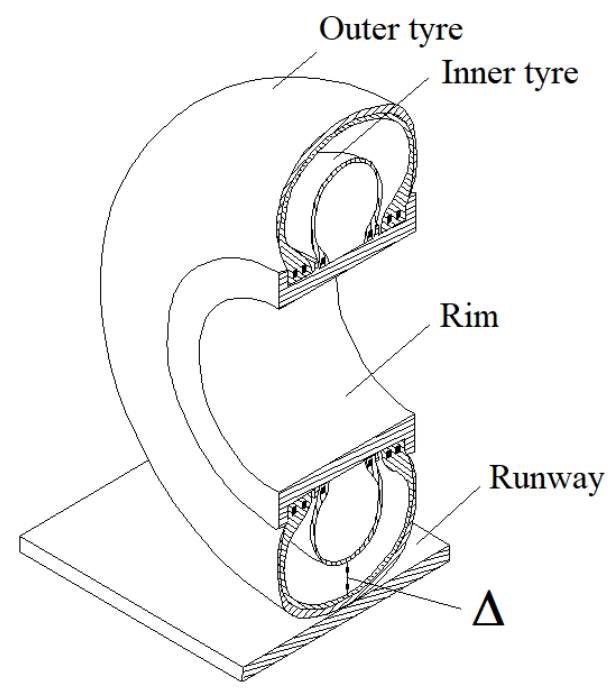

Fig. 3. Distance between inner and outer tire $\Delta$
Another role of the smaller (inner) tire is to receive kinetic energy on its own, the result of which is that the outer tire has reduced overpressure of $50 \%$ compared to the standard value. In this way, the durability of the outer tire is increased in the proposed construction solution. This is a different approach from [13], in which it was proposed to turn the wheel before touching the runway to increase its durability. In addition to extending the life of the tires, reducing the tires' overpressure also contributes to the durability of the runway surface [14].

\section{ANALYSIS}

The analysis of behaviour of the proposed set of tires (Fig. 3) when absorbing the kinetic energy during the contact of the outer tire with a runway is a complex problem, which is reflected in geometric and material nonlinearity. It also implies large deformations and indeterminate loading conditions to which the set of tires is exposed. The complexity is not reflected only in the undefined conditions that occur between the tires and wheels, tires, and runway, but also in their interaction, i.e., the contact of the outer torus of the smaller tire and the inner torus of the larger tire.

Numerical analysis of the proposed design solution is performed as an implicit simulation by using the software package ANSYS. The performed implicit numerical analysis of aircraft tires takes into consideration the following problems: the definition of conditions and loads, geometry definition, and material definition.

\section{DEFINITION OF CONDITIONS AND LOADS}

In addition to the indeterminate conditions that occur between the outer tire and the rim, the outer tire, and the runway [15], in the new construction solution, there are also indeterminate conditions between the outer and inner tires. These indeterminate conditions are defined as contact problems:

- The contact problem between the outer tire and the rim with a coefficient of friction $\mu=0.59$ [16], as there is no slip between the tire and the rim;

- Contact problem between the outer torus of the inner tire and the inner torus of the outer tire with a static friction coefficient $\mu=1.16$ [17];

- Contact problem between the outer tire and the runway with friction coefficient $\mu=0.3$ [16].

The contact between the outer (standard) tire and the rim is defined as contact problem, while the inner (smaller) tire is placed such that the displacement of its contact surface (with the rim) in all directions is 
disabled. More precisely, the moving of the nodes on the contact surface of the inner tire with the rim was disabled in the model. These constraints were imposed for practical reasons, i.e., this enables the use of the models previously designed and analysed for standard tires.

In real-life situations, a tire impacts the runway, which is stationary. However, in this simulation, the opposite approach was used: the tire is fixed (the displacements towards the $x, y$, and $z$ directions take the value of 0 ), while the runway (surface) is exerting the load. The load on the tire is exerted by the surface (runway) such that the points of force applications are placed at the edges of the surface in the model. The force in which the tire exerts the surface is divided by four, and these four parts of the force are taken as inputs in the model on the considered key points of the surface. As we are dealing with a symmetric model, it was sufficient to take only two out of these four parts of the total force.

The set of tires is exposed to the pressure, which is the result of the pressure of the tire walls surface (to the internal torus of the outer tire and to the internal and external toruses of the inner tire). In the conducted analysis, the pressure of $p_{0}=0.2 \mathrm{MPa}$ was applied to the inner torus of the outer tire and the inner and outer torus of the inner tire, which is half of the regular pressure of the standard (outer) tire without inner tire inside. Without this initial step, the tires would not have the necessary stiffness effect caused by the air pressure.

Because the deformation process is very fast, the process is considered to be adiabatic, differently from [11], where the change of temperature occurs.

\section{DEFINITION OF GEOMETRY AND MATERIALS}

The shape of the tire was modelled based on the geometry obtained by scanning the cross-section of a tire name size $23 \times 7.2-10$. This tire was physically cut to obtain the precise shape of the cross-section. Starting from the scanned cross-section, we have performed its decomposition to constructive elements: carcass, tread, and bead, as shown in Fig. 4.

This decomposition showed to be the most adequate, as it leads to easier geometry modelling, defining the material, and creating the mesh of finite elements in the numerical software. In addition, such decomposition enables analysing the whole assembly design, as well as the stress-strain analysis of each constructive element of the tire separately (carcass, protector, bead, rim, and the runway).

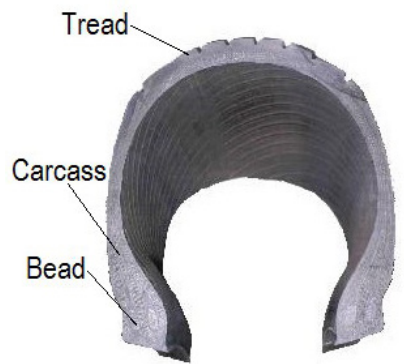

Fig. 4. The aircraft tire cross-section

The dimensions of the smaller tire were defined as $18.3 \times 3.7-10$. The geometry of the smaller-inner tires is modelled through the basic structural elements.

The tread pattern of the standard (outer) tire was not modelled in order to avoid the large number of finite elements in the pattern. As the goal of the analysis is the behaviour of the set of tires during symmetrical landing, the analysis is focused on the carcass, not on the tread. Therefore, this simplification of the tread will not lead to significant errors in the analysis.

In this study, the finite elements mesh consisting of 18,258 elements of the type SOLID185 was applied (Fig. 5). To achieve mesh consistency, only one element type was used. SOLID185 was chosen, because it gives the possibility of modelling both solid and layered structures. It is available in two forms [18]: standard (nonlayered) structural solid (SOLID185 - 3-D 8-Node Structural Solid) and layered structural solid (SOLID185 - 3-D 8-Node Layered Solid). Therefore, the tread and rim were modelled as structural solid, while carcass was modelled ad layered solid. Cord in carcass is defined by using shell section in combination with SOLID185 - 3-D 8-Node Layered Solid.

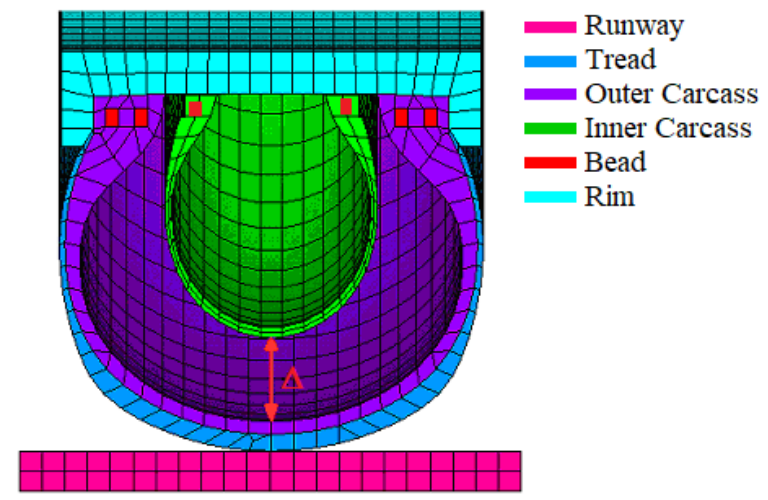

Fig. 5. FE model of set of tires with constituents components

In the analysis, bead, rim, and the runway were modelled as isotropic materials, by defining their 
characteristics, specifically Young`s modulus of elasticity and Poisson's ratio (see Table 1).

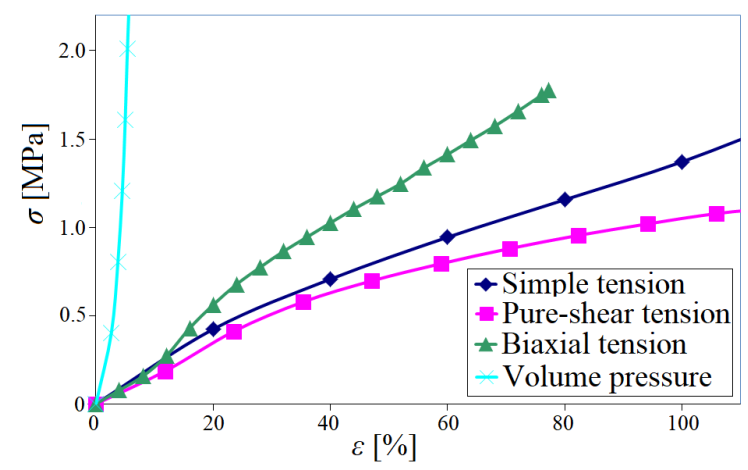

Fig. 6. Experimentally obtained Stress-strain curves

The larger and smaller tires carcasses are defined as an anisotropic material consisting of several layers of cord. We have obtained mechanical characteristics of carcasses by performing a tensile test in the direction of the warp and fill (see Table 1).

Table 1. Characteristics of materials

\begin{tabular}{|c|c|c|c|c|}
\hline & & $\begin{array}{l}\text { Poisson } \\
\text { ratio, } v\end{array}$ & $\begin{array}{c}\text { Young's modulus, } \\
E \text { [daN/mm²] }\end{array}$ & $\begin{array}{c}\text { Shear modulus, } \\
G \text { [daN/mm²] }\end{array}$ \\
\hline Bead & & 0.30 & 21000 & \\
\hline Rim & & 0.30 & 7390 & \\
\hline \multirow{2}{*}{ Cord } & Warp & 0.35 & 210 & 74 \\
\hline & Fill & 0.35 & 71 & 26 \\
\hline
\end{tabular}

The Mooney-Rivlin first-order material model, (in the literature known as "the two-parameter Mooney-Rivlin material model") was used to model the hyperelastic material. According to the model, the strain energy potential $(W)$ is defined as the sum of isochoric deformation energy $\left(W^{i s o}\right)$ and volume deformation energy $\left(W^{v o l}\right)$ :

$$
W=W^{i s o}+W^{v o l} .
$$

The function of isochoric deformation energy is given in the following form [19]:

$$
W^{i s o}=c_{10}\left(I_{1}-3\right)+c_{01}\left(I_{2}-3\right),
$$

while the function of volume deformation energy is defined as follows:

$$
W^{v o l}=\frac{1}{d}\left(\lambda_{1} \lambda_{2} \lambda_{3}-1\right)^{2}=\frac{1}{d}(J-1)^{2} .
$$

The value of incompressibility material parameter $d$ is obtained as:

$$
d=\frac{(1-2 v)}{c_{10}+c_{01}}
$$

Finally, according to the Mooney-Rivlin model [20], the expression for the strain energy potential of an incompressible hyper-elastic material can be written as follows:

$$
W^{i s o}=c_{10}\left(I_{1}-3\right)+c_{01}\left(I_{2}-3\right)+\frac{1}{d}(J-1)^{2} .
$$

The values of Mooney-Rivlin coefficients $c_{10}$, $c_{01}$, and $d$ are calculated by numerical software with experimentally obtained input data. Bearing in mind that the rubber performs differently as a material [21] during tensioning, compression, as well as being incompressible, in this study, the following experiments were carried out [22]: tensioning [23], pure shear [24], biaxial tensioning [25], and volume compression. The obtained experimental data of the stress-strain performance of the material are shown in Fig. 6. These results were used in numerical software to determine Mooney-Rivlin coefficients and to model the tread as hyperelastic material (see Table 2).

Table 2. Mooney-Rivlin model characteristics

\begin{tabular}{lccc}
\hline \multirow{2}{*}{ Tread } & $c_{01}[\mathrm{MPa}]$ & $c_{10}[\mathrm{MPa}]$ & $d[1 / \mathrm{MPa}]$ \\
\cline { 2 - 4 } & 0.0672 & 0.034298 & 27.408 \\
\hline
\end{tabular}

\section{RESULTS AND DISCUSSION}

Fig. 7 shows the change in the cross-sectional area of the set of tires. If we compare the deformation of the outer tire (Fig. 1) with this deformation, it can be noticed that the contact surface of the set of tires set remains flat with the runway surface. In other words, there is no drawing inward of the outer tire, due to the deflection at the moment of its contact with the runway.

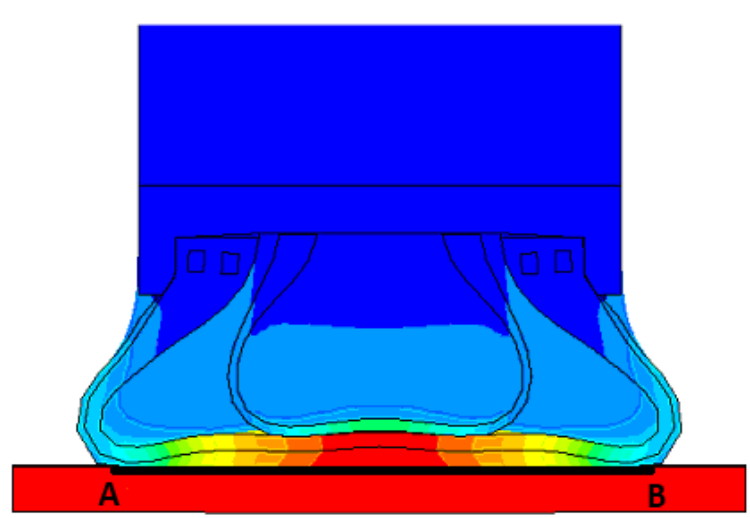

Fig. 7. Cross-section of the set of tires at deflection $h=70 \mathrm{~mm}$; $A, B$ the outer boundaries of the contact surface 
The same can be observed in the longitudinal section of the set of tires (Fig. 8). Fig. 2 shows how the outer tire is drawing inward in the longitudinal section, due to the deflection at the moment of its contact with the runway. In Fig. 8, it can be seen that the inner tire does not allow the outer tire to draw inward, and thus, forces it to expand laterally, increasing its track width.

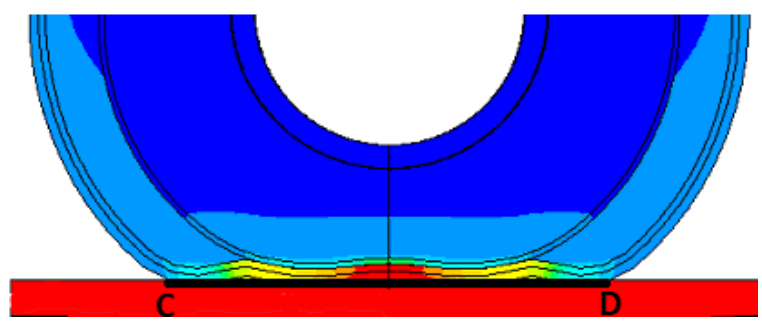

Fig. 8. Longitudinal section of the set of tires at deflection $h=70$ $\mathrm{mm} ; \mathrm{C}, \mathrm{D}$ the outer boundaries of the contact surface

The diagram in Fig. 9 presents the tire deflection with respect to the contact force of tires and the runway. It can be observed that for the same force, the deflection of the tire set is greater than the deflection of the standard tire for $h<40 \mathrm{~mm}$. This happens because the overpressure in the set of tires is two times smaller compared to the overpressure in the standard tire. On average, the deflection of the set of tires for $h<40 \mathrm{~mm}$ is around $10 \%$ larger compared to the deflection of the standard tire.

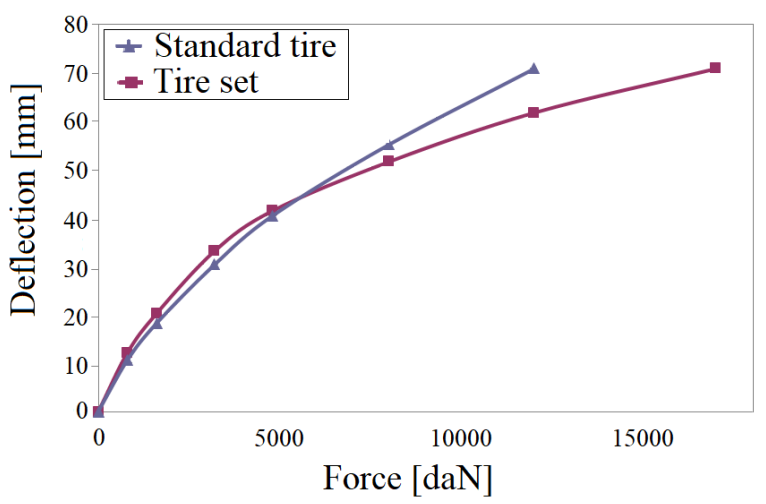

Fig. 9. Diagram of change of tire deflection on $A-B$ in respect to the contact force of tire and runaway

From the moment of contact of the outer and inner tire $(h>40 \mathrm{~mm})$ for the same contact force of tire and runway, the deflection of the set of tires is much smaller than the deflection of a standard tire. If we consider the intensity of the force when the deflection of a standard tire is equal to $h=70 \mathrm{~mm}$, it can be observed that the deflection of the set of tires is around $15 \%$ smaller than in the case of a standard tire.
In addition, it can be noticed that the force required to reach the deflection $h=70 \mathrm{~mm}$ is $67 \%$ greater in the case of a set of tires compared to a standard tire.

The diagram in Fig. 10 shows the dependence of the tire track width from the deflection on part A-B. It can be observed that for the same tire deflection (up to $h<40 \mathrm{~mm}$ ), the tire track width is larger for a standard tire than for the set of tires. This is the result of lateral expansion caused by deflection and overpressure that is two times higher in a standard tire compared to the overpressure in the set of tires.

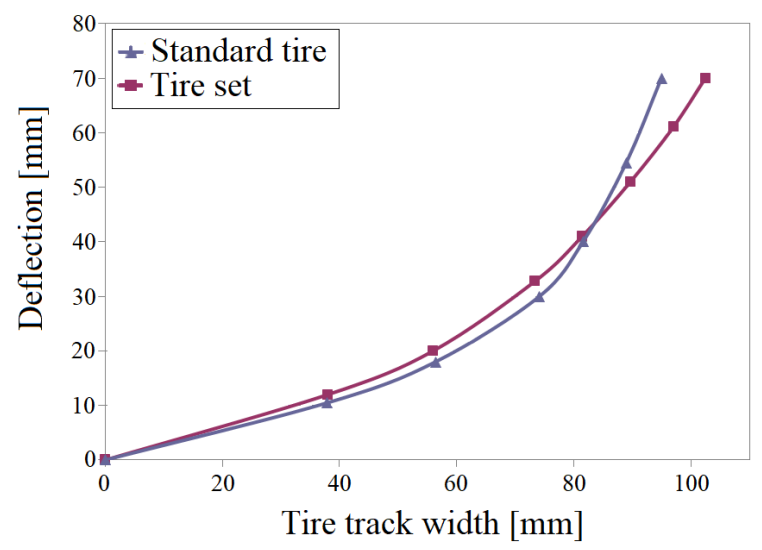

Fig. 10. Diagram of change of tire track width on section $A-B$ with respect to tire deflection

From the moment of contact of the outer and inner tire $(h>40 \mathrm{~mm})$, as the deflection increases, the track width of the set of tires is larger than in the track width of a standard tire. Thus, at the final deflection $h=70 \mathrm{~mm}$, the track width of a set of tires is $8 \%$ larger compared to the case of a standard tire. This is because the inner tire does not allow the outer tire to be drawn inward, and consequently, the outer tire is extending laterally (Fig. 7).

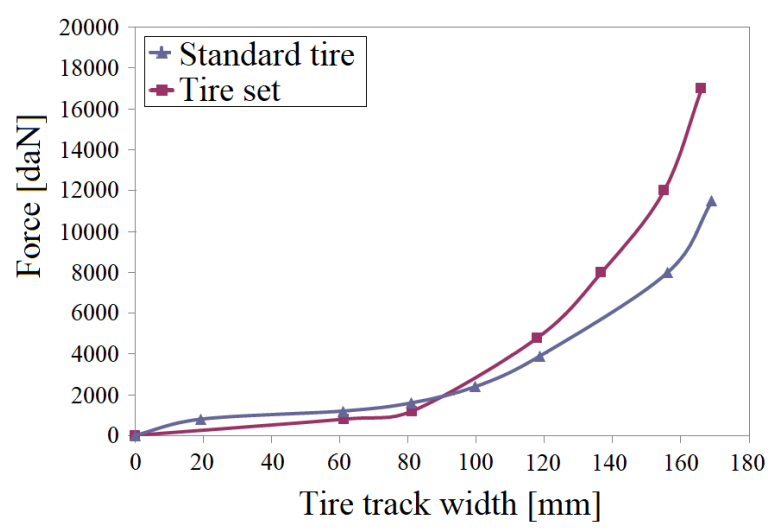

Fig. 11. Diagram of change of tire track width on C-D section in respect to the landing force 
The diagram in Fig. 11 presents the changes of the tire track width on section C-D in respect to the landing force. It can be observed that for the deflection of $h<40 \mathrm{~mm}$, the tire track width is almost the same for standard tire and the set of tires. At the deflection of $h=70 \mathrm{~mm}$, it can be noticed that for the same tires track width in section C-D, the absorption of the set of tires is around $67 \%$ larger compared to a standard tire.

The diagram of Fig. 12 shows the results of the stress analysis in the carcass for deflection of $h=70$ $\mathrm{mm}$ on section A-B below the tread for both standard tire and the set of tires. It can be observed that the stress in the carcass is around $37 \%$ lower in the set of tires compared to the standard tire. In addition, the stress in the carcass sections A-E and B-F below the tread (which is in contact with the runway) are the same for both standard tire and the set of tires.

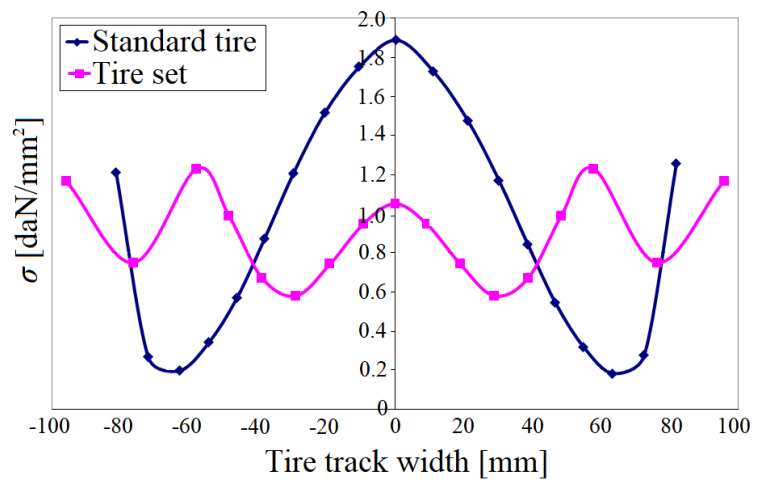

Fig. 12. Diagram of the distribution of von Mises stresses in the carcass below the tread for $h=70 \mathrm{~mm}$, in section $A-B$

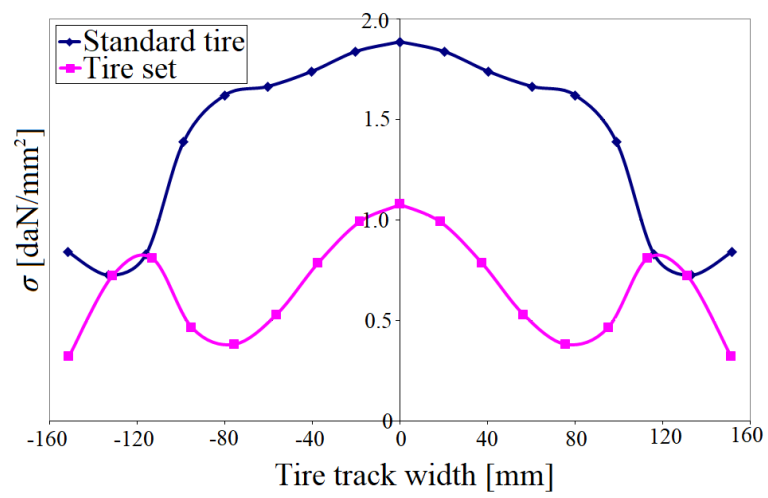

Fig. 13. Diagram of the distribution of von Mises stresses in the carcass below the tread for $h=70 \mathrm{~mm}$, in the section $C-D$

The diagram of Fig. 13 gives the results of the stress analysis in the carcass section C-D below the tread for both standard tire and the set of tires, for the deflection of $h=70 \mathrm{~mm}$. It can be seen that the stress in the carcass is $42 \%$ lower in the case of the set of tires than in the standard tire. Furthermore, it can be observed that the stresses in the carcass sections $\mathrm{C}-\mathrm{G}$ and D-H below the tread (which is in contact with the base) are the same for both standard tire and the set of tires. In contrast, the load-capacity of the set of tires is approximately $56 \%$ higher compared to the loadcapacity of a standard tire (for deflection $h=70 \mathrm{~mm}$ ).

\section{CONCLUSION}

This study presents a comparative analysis of stressstrain behaviour of a standard tire and the new constructive solution of the set of tires. The analysis was conducted for different aircraft landing forces by using finite elements. It was shown that the problem of analysing aircraft tires for different aircraft inclinations requires highly complex modelling procedure for both model and material.

The analysis conducted in this study aimed to investigate the possibility of reducing the pressure in the tires while preserving its dimensions and the kinetic energy absorption. The change in tire geometry during landing at the time of direct contact of the tires with the runway at maximum tire deflection up to $h$ $=70 \mathrm{~mm}$ was analysed by comparing the results for a standard (outer) tires and a set of tires consisting of an outer (standard) and a smaller (inner) tire. The set of tires was exposed to the overpressure that was two times lower compared to the overpressure in the standard tire.

Based on the results of comparative analyses, it can be concluded that the new technical solution leads to the increase of the tire tread width by $8 \%$, and therefore, it ensures a larger contact area between the tire and the runway. It results in the fact that the tire stress is approximately $40 \%$ lower for the tire set compared to a standard tire, which further leads to higher durability of the tire in a new solution, as well as the ability to absorb around $56 \%$ more kinetic energy than in the case of a standard tire.

\section{NOMENCLATURE}

$W \quad$ strain energy potential function, [MPa]

$W^{i s o}$ isochoric deformation energy function, [MPa]

$W^{\text {vol }}$ volume deformation energy function, [MPa]

$\lambda_{1}, \lambda_{2}, \lambda_{3}$ principal stretch ratios,

$I_{1}, I_{2} \quad$ principal deviatory strain invariants,

d material incompressibility parameter, [1/MPa]

$v \quad$ Poisson ratio 
$c_{10}, c_{01}$ materials constants, [MPa]

$J \quad$ volume ratio, $J=\lambda_{1} \lambda_{2} \lambda_{3}$

$\Delta \quad$ distance between the inner and outer tire, [mm]

$\mu \quad$ friction coefficient

$h \quad$ deflection of a tire, $[\mathrm{mm}]$

$p_{0} \quad$ initial pressure, $[\mathrm{MPa}]$

E Young's modulus, [daN/mm²]

$G \quad$ Shear modulus, [daN $\left./ \mathrm{mm}^{2}\right]$

\section{REFERENCES}

[1] Aircraft Tire Care and Maintenance Manual, from www. goodyearaviation.com, accessed on 2019-06-11, Goodyear, Akron.

[2] Wang, W., Zhao, Y., Wang, J., Zang, L. (2013). Structure analysis and ride comfort of vehicle on new mechanical elastic tire. Proceedings of the FISITA 2012 World Automotive Congress, Lecture Notes in Electrical Engineering, vol. 198, p. 199-209, Dol:10.1007/978-3-642-33795-6_17.

[3] Guo, H., Bastien, C., Blundell, V.M., Wud, G., (2013). A detailed aircraft tyre finite element model for hard landing safety assessment. 9th European LS-DYNA Conference, Manchester.

[4] Essienubong, I.A., Ikechukwu, O., Paul, S. (2018). Finite element analysis of aircraft tire behaviour under overloaded aircraft landing phase. Aeronautics and Aerospace Open Access Journal, vol. 2, no. 1, p. 32-37, D0l:10.15406/ aaoaj.2018.02.00026.

[5] Behroozi, M., Olatunbosun, O.A., Ding, W. (2012). Finite element analysis of aircraft tyre - Effect of model complexity on tyre performance characteristics. Materials and Design, vol. 35, p. 810-819, Dol:10.1016/j.matdes.2011.05.055.

[6] Kongo Kondé, A., Rosu, I., Lebon, F., Brardo, O., Devésa, B. (2013). On the modeling of aircraft tire. Aerospace Science and Technology, vol. 27, no. 1, p. 67-75, Dol:10.1016/j. ast.2012.06.008.

[7] Korunović, N., Trajanović, M., Stojković, M. (2007). FEA of tyres subjected to static loading. Journal of the Serbian Society for Computational Mechanics, vol. 1, no. 1, p. 87-98.

[8] Wood, G., Blundell, M., Sharma, S. (2012). A low parameter tyre model for aircraft ground dynamic simulation, Materials and Design, vol. 35, p. 820-832, D0l:10.1016/j. matdes.2011.03.041.

[9] Birembaux, L.E., Rosu, I., Lebon, F. (2013). Thermo-mechanical modelling of the aircraft tyre cornering. Machine Dynamics Research, Warsaw University of Technology edition, vol. 37, no. 1, p. 29-36.

[10] Lin, Y.-J., Hwang, S.-J. (2004). Temperature prediction of rolling tires by computer simulation. Mathematics and Computers in Simulation, vol. 67, no. 3, p. 235-249, D0I:10.1016/j. matcom.2004.07.002.

[11] Kongo-Kondé, A., Rosu, I., Lebon, F., Brardo, O., Devésa, B. (2013). Thermomechanical analysis of an aircraft tire in cornering using coupled ale and lagrangian formulations.
Central European Journal of Engineering, vol. 3, no. 2, p.191205, DOI:10.2478/s13531-012-0049-6.

[12] Baranowski, P., Bogusz, P., Gotowicki, P., Malachowski, J. (2012). Assessment of mechanical properties of offroad vehicle tire: Coupons testing and FE model development. Acta Mechanica et Automatica, vol. 6, no. 2, p. 17-22.

[13] Alroqi, A.A., Wang, W.J. (2016). Reduction of aircraft tyre wear by pre-rotating wheel using ANSYS Mechanical Transient. Advanced Engineering Forum, vol. 17, p. 89-100, DOI:10.4028/www.scientific.net/AEF.17.89.

[14] White, G. (2017). Limitations and potential improvement of the aircraft pavement strength rating system to protect airport asphalt surfaces. International Journal of Pavement Engineering, vol. 18, no. 12, p. 1111-1121, Dol:10.1080/102 98436.2016.1155122.

[15] Korunović, N., Trajanović, M., Stojković, M., Mišić, D., Milovanović, J. (2011). Finite element analysis of a tire steady rolling on the drum and comparison with experiment. Strojniški vestnik - Journal of Mechanical Engineering, vol. 57, no. 12, 888-897, Dol:10.5545/sv-jme.2011.124.

[16] Radonjić, S., Baralić, J., Dučić, N. (2011). Determination of static friction factor by using tribometer. Technology, Informatics and Education for Learning and Knowledge Society, $6^{\text {th }}$ International Symposium, Technical Faculty Čačak.

[17] Engineering ToolBox (2004). Friction and Friction Coefficients.

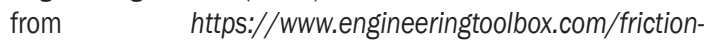
coefficients-d_778.html, accessed 2020-05-15.

[18] Ansys (2012). Theory Reference, Release 14.5, ANSYS Inc. Canonsburg.

[19] Kumar, N., Rao, V.V. (2016). Hyperelastic Mooney-Rivlin model: Determination and physical interpretation of material constants. MIT International Journal of Mechanical Engineering, vol. 6, no. 1, p. 43-46.

[20] Shahzad, M., Kamran, A., Siddiqui, M.Z., Farhan, M. (2015). Mechanical characterization and FE modelling of a hyperelastic material. Materials Research, vol. 18, no. 5, p. 918-924, DOl:10.1590/1516-1439.320414.

[21] Ogden, R., Saccomandi, G., Sgura, I. (2004). Fitting hyperelastic models to experimental data. Computational Mechanics, vol. 34, p. 484-502, D0l:10.1007/s00466-0040593-y.

[22] Jakel, R. (2010). Analysis of Hyperelastic Materials with MECHANICA - Theory and Application Examples. PTC Presentation for the $2^{\text {nd }}$ SAXSIM.

[23] Jadhav, A. N., Bahulikar, S.R., Sapate, N.H. (2016). Comparative study of variation of mooney-rivlin hyperelastic material models under uniaxial tensile loading. International Journal of Advance Research and Innovative Ideas in Education, vol. 2, no. 4, p. 212-216.

[24] Keerthiwansa, R., Javorik, J., Kledrowetz, J., Nekoksa, P. (2018). Elastomer testing: The risk of using only uniaxial data for fitting the Mooney-Rivlin hyperelastic-material model. Materials and Technology, vol. 52, no. 1, p. 3-8, Dol:10.17222/ mit.2017.085.

[25] Duncan, C.B., Crocker, L.E., Maxwell, A., Hunt, R. (1999). Verification of Hyperelastic Test Methods. Technical Report, National Physical Laboratory Teddington, Middlesex. 